\title{
(6) OPEN ACCESS \\ Acknowledging awareness: informing families of individual research results for patients in the vegetative state
}

\author{
Mackenzie Graham, ${ }^{1}$ Charles Weijer, ${ }^{1,2}$ Andrew Peterson, ${ }^{1,2}$ Lorina Naci, ${ }^{2}$ \\ Damian Cruse, ${ }^{2}$ Davinia Fernández-Espejo, ${ }^{2}$ Laura Gonzalez-Lara, ${ }^{2}$ Adrian M Owen ${ }^{1,2}$
}

${ }^{1}$ Department of Philosophy, Rotman Institute of Philosophy, Western University, London, Ontario, Canada ${ }^{2}$ Department of Psychology, The Brain and Mind Institute, Western University, London, Ontario, Canada

\section{Correspondence to} Dr Charles Weijer, Rotman Institute of Philosophy,

Western University, 1151 Richmond Street, London, ON, Canada N6A 5B8; cweijer@uwo.ca

Received 6 February 2014 Revised 3 July 2014 Accepted 15 July 2014 Published Online First 30 July 2014

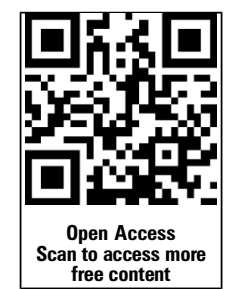

\section{ABSTRACT}

Recent findings in cognitive neuroscience have revealed that some patients previously diagnosed as being in a vegetative state may retain some degree of covert awareness. However, it is unclear whether such findings should be disclosed to the families of these patients. Concerns about the preservation of scientific validity, reliability of results and potential harms associated with disclosure suggest that individual research results should be disclosed only under certain conditions. In the following paper, we offer four criteria for the disclosure of individual research results. Because the results of functional neuroimaging studies to detect covert awareness in vegetative patients are scientifically valid, informative and reasonably reliable and have considerable potential benefit for the patient, researchers have an obligation to disclose such results to family members. Further work is needed to develop educational materials for families and to systematically study the impact of disclosure on the families themselves.

Imagine the following scenario. John was a healthy and active 18 -year-old man, who suffered a severe head injury in a motor-vehicle accident. After days in a coma, John emerged into a vegetative state, in which he was awake but showed no signs of awareness of himself or his environment.

Five years have passed since John's injury. Since returning home, John has been assessed dozens of times by experienced neurologists; each time, the standardised behavioural assessments confirmed the diagnosis that he was vegetative. Recently, John's attending physician informed John's family about a research project in which neuroscientists use functional MRI to look for evidence of awareness that behavioural assessments might have missed. While his family has come to accept that a full recovery is unlikely, they continue to believe that John has some awareness, and are eager to find out any information they can about John's mental life (Does he recognise them? Can he understand them? Is he suffering?). They also believe that rehabilitation might improve John's condition, and that, if it can be confirmed that he is aware, he might be given more access to these resources. John's family consents on his behalf to participate in the neuroimaging study. What information-if any-should researchers disclose to John's family?

There has been considerable debate in recent years regarding the obligation of researchers to disclose their findings to study participants. Most commentators agree that summary research results - results that address the study hypothesis across a research population-ought to be provided to participants upon completion of the study. ${ }^{1-3}$ However, questions remain as to whether researchers have an obligation to disclose individual research results-results that address the study hypothesis in a particular participant. Considerations include the potential benefit and harm of disclosure, as well as the impact of disclosure on the scientific validity and reliability of results. ${ }^{4} 5$ This paper examines the disclosure of individual research results in a novel context: the use of functional MRI to detect covert awareness in vegetative patients. While research investigating awareness in patients with severe brain injury is currently underway in a number of locations, ${ }^{6} 7$ this paper will focus on work being conducted by five of the authors (AMO, LN, DC, DF-E, LG-L) and colleagues at Western University's Brain and Mind Institute in London, Ontario.

\section{DISCLOSURE OF INDIVIDUAL RESEARCH RESULTS}

Most existing ethical guidelines justify disclosure based on the ethical principle of beneficence, and set a high threshold for disclosing individual research results. Research results that are unreliable (ie, have not been replicated in subsequent studies) or meaningless (ie, lack utility) are of little value to research participants, may cause unnecessary anxiety, and may lead participants to make illadvised medical decisions. ${ }^{8-10}$ Because the goal of research is to generate knowledge for the benefit of society, disclosing individual results contradicts this aim if it compromises scientific validity. This goal may be over-ridden, however, when the information to be disclosed has sufficient potential benefit to the participant. Accordingly, most guidelines stipulate that research results must be clinically useful if they are to be disclosed to participants. For example, the US National Bioethics Advisory Commission states that disclosure should occur only when '(a) findings are scientifically valid and confirmed, (b) findings have significant implications for the subject's health concerns, and (c) a course of action to treat or ameliorate these concerns is readily available'. 8 
Critics of these guidelines reject the notion that disclosure should be limited to results that have clinical utility or that relate to conditions for which no treatment exists and argue that respect for persons generates an obligation to make individual research results available to participants. ${ }^{3}$ Accordingly, much of the debate in this domain has centred on extending the threshold for disclosure, to include results that have personal utility (eg, those that might influence life-planning or reproductive decision-making) or that might otherwise be meaningful to participants.

We argue that researchers have an obligation to disclose individual results to participants when doing so is consistent with both the ethical principles of beneficence and respect for persons. Beneficence requires that researchers minimise the potential harms and maximise the potential benefits of their research, and ensure that the potential benefits of the research (both to the participant directly and to society) are reasonably balanced in relation to the potential harms of research.

Respect for persons requires that researchers respect the autonomy of decisionally capable individuals, and protect those with developing, impaired, or diminished autonomy. A researcher satisfies this obligation by ensuring that participants are informed about the research, and allowing them to make decisions regarding participation based on their own values and preferences. In cases in which research participants are incapable of exercising autonomy, researchers show respect for them by appealing to a proxy decision-maker. The proxy decision-maker is charged with making an informed decision on behalf of the participant, based on either his or her expressed wishes or best interests.

Given these principles, we present four criteria for the disclosure of individual research results. Satisfaction of these criteria is sufficient to justify disclosure.

1. Disclosure does not undermine the scientific validity of the study.

2. The results are informative and reliable.

3. The potential benefits of disclosure to the participant outweigh the potential harms.

4. The participant consents to be informed of the results.

Before applying these criteria to the question posed at the outset, we provide some relevant background information on the vegetative state, the mental imagery task used to assess covert awareness, and the individuals participating in this research.

\section{VEGETATIVE STATE}

In the context of clinical neurology, consciousness is typically understood as consisting of two components: 'wakefulness (also known as arousal, referring to the level of consciousness) and awareness (the contents of consciousness)'. ${ }^{11}$ Wakefulness is a state in which the eyes are open and may be accompanied by a degree of motor arousal; awareness, meanwhile, entails having certain experiences. While patients in the vegetative state may demonstrate wakefulness, they are unable to voluntarily (ie, nonreflexively) respond to visual, auditory, tactile or noxious stimuli and fail to provide evidence of language comprehension or meaningful expression. ${ }^{12}$ It is on this basis that awareness is assumed to be absent. Accordingly, the vegetative state is often referred to as 'wakefulness without awareness'. The vegetative state can be caused by 'acute traumatic and non-traumatic brain injuries, degenerative and metabolic brain disorders, as well as severe congenital malformations in the nervous system'. ${ }^{12}$ Recovery of consciousness is unlikely after 12 months if the vegetative state is caused by brain trauma, and rare after 3 months if it is caused by anoxia or other brain disorders. The life expectancy of patients in the vegetative state is between 2 and 5 years, although survival beyond 15 years is not unheard of. ${ }^{13}$

The prevalence of the vegetative state in adults is between 40 and 168 per million in the USA, although precise epidemiological data are lacking. ${ }^{14}$ This is due in part to the fact that the clinical assessment of vegetative patients is based on subjective interpretation of inconsistent behaviours in patients with severely limited motor skills. ${ }^{15}$ It is possible that a patient who is aware, but behaviourally non-responsive, might be diagnosed as vegetative. In fact, several studies have shown that as many as $40 \%$ of patients diagnosed as vegetative actually possess at least minimal responsivity and awareness. ${ }^{16-18}$ Moreover, of the $60 \%$ who are correctly diagnosed as vegetative on the basis of behavioural diagnostic criteria, an estimated 17\% retain covert awareness undetectable through routine clinical assessment. ${ }^{19}$

\section{MENTAL IMAGERY TASK}

As neuroimaging has become increasingly sophisticated, new techniques have emerged to supplement traditional, bedside assessment methods. One technique used to detect covert awareness has been successfully developed. ${ }^{19}$ Study participants are placed in a functional MRI scanner and repeatedly instructed to imagine playing tennis or walking from room to room in their house for $30 \mathrm{~s}$ intervals. These tasks have been consistently and reliably shown to activate distinct areas of the brain in $100 \%$ of healthy volunteers. ${ }^{20}$ Successful activation of these areas in response to the researcher's commands reveals that these participants retain the capacity for sustained attention (required to maintain focus), language comprehension (required to understand instructions), response selection (required to switch between alternative tasks or conditions) and working memory (required to remember which task to perform when instructed).

The ingenuity of this technique stems partly from the fact that it relies on an established model of awareness assessment and translates it to a new modality. ${ }^{21}$ In the absence of specific neural markers, the only way in which an individual can demonstrate awareness is by responding to commands through some form of behaviour; this is true of both healthy individuals and those with disorders of consciousness. Given that patients in the vegetative state are behaviourally non-responsive, the mental imagery task provides an alternative mode by which they can demonstrate command-following abilities and thereby indicate awareness.

Researchers have also employed a modified version of the mental imagery task to successfully communicate with participants diagnosed as vegetative. In two cases, participants imagined playing tennis to indicate 'yes' and imagined walking from room to room in their house to indicate 'no' in response to externally verifiable questions, including their name, the current date, and the name of their personal support worker. ${ }^{19} 22$

Both the mental imagery task and its modified versions yield robust results. Successful completion allows researchers to be highly confident that the participant possesses awareness. However, if the participant is unable to complete the task, it is virtually impossible for researchers to determine whether he or she lacks awareness or if there was a failure in the test conditions. This may occur if the participant fails to understand the instructions, falls asleep during the test, or moves frequently, thereby preventing researchers from obtaining images suitable for analysis. ${ }^{23}$ 


\section{PARTICIPANT POPULATION AND FAMILIES}

Since 2012, 24 individuals with an average age of 35.2 years (range 18-60 years) have participated in some form of mental imagery task. Of this participant group, 14 were diagnosed as vegetative, 9 as minimally conscious, and 1 as having locked-in syndrome. The median time since injury was 5.0 years (range 1-19 years); 11 of the participants had suffered traumatic brain injury; 8 were living at home; the remaining 16 were being cared for in hospital. Most participants had already undergone MRI or CT imaging and repeated behavioural tests to assess awareness. In all cases, the severity of the patient's neurological condition requires that the decision to participate in research and to be informed of results is made by the patient's family.

The families have discussed the participant's diagnosis and prognosis with a neurologist. Yet, few studies have examined the needs and attitudes of these families. In one study, Tresch and colleagues interviewed 33 family members of vegetative patients (ranging from 12 to 204 months after the injury with a mean duration of 54 months) and found that $90 \%$ of them believed the patient had some awareness of pain, environment, taste, conversation, or the presence of others. However, 32 of the 33 family members did not expect the patient to improve. ${ }^{24}$ Families also express a strong desire to be informed by physicians about what is done to their family member and to be involved in decision-making. The experiences in this study are consistent with Tresch's results; while family members commonly believe that the participant remains aware on some level, they have come to accept his or her condition and do not anticipate recovery.

\section{DISCLOSURE OF INDIVIDUAL RESEARCH RESULTS IN A DISORDER OF CONSCIOUSNESS CONTEXT}

What action guidance do our proposed criteria provide for researchers regarding the disclosure of individual research results in this study context? In the case of positive results, the four criteria we have identified above clearly support disclosure.

\section{Disclosure is consistent with scientific validity}

One of the primary concerns with disclosing individual research results is that doing so compromises scientific validity and deprives society of the potential benefits that the knowledge generated by research might provide. While most functional MRI studies average results across a group of participants, studies involving participants with disorders of consciousness differ in that they generally seek to establish whether the individual is aware. While participants may be scanned as part of a convenience sample, each participant forms a discrete research study because the results obtained from one participant do not affect the results of another. Further, because it occurs after the completion of the study, disclosure does not affect the researcher's interpretation of the results, nor does it compromise the research protocol (affect scientific validity, and potential benefits to society). Disclosing individual research results thus satisfies the first criterion.

\section{Results are informative and reliable}

The experimental procedure used has been refined over the course of many functional MRI sessions with healthy controls ${ }^{20}$ and is based on a well-established model of awareness assessment, namely, command-following. This allows the research team to be highly confident of the presence of awareness given successful completion of the study tasks. This mitigates concerns that observed responses might simply be automatic. ${ }^{25}$
Furthermore, because a positive result means that the participant retains the ability to respond to instructions and exhibit willed, voluntary behaviour, these results are informative both for researchers and families. Indeed, such information may change a family's decision-making or provide further information to physicians as they formulate a comprehensive picture of the participant's condition. Disclosing individual results in this context is thus consistent with the principle of respect for persons, and satisfies the second criterion.

\section{Potential benefits of disclosure outweigh potential harms for participants}

The most substantial benefit associated with the disclosure of individual research results accrues to the participants themselves. If the participant is aware, it is beneficial that this is acknowledged. A positive result on the mental imagery task entails that the participant possesses awareness, characterised by auditory processing, language comprehension, short-term memory, and executive function. The knowledge that the participant is aware is likely to alter how others interact with him or her and may contribute to an increased sense of personhood. Kitwood argues that personhood consists of one's 'experiential self', which arises out of interacting with others in a context of mutual respect. ${ }^{26}$ Although vegetative patients may not be capable of communicating directly with others, the knowledge that they are aware allows others to recognise their personhood, and interact with them as persons worthy of respect and consideration. As Martin Buber perceptively notes, acknowledging a person's awareness causes us to treat him or her as a 'you' rather than an 'it'. ${ }^{27}$

Moreover, knowledge that the participant is aware may alter the manner in which he or she receives ongoing treatment (eg, a more careful use of analgesics if the participant is sentient), or justify more treatment (eg, more physiotherapy). While we do not suggest that patients in the vegetative state receive anything less than proper care, vegetative patients are, by definition, thought to be incapable of any experience whatsoever, including pain, which may be seen as a justification for withholding analgesics. $^{28}$ The discovery of awareness in study participants provides an imperative for treating them with the utmost care, because they may be sentient.

It may also justify further attempts to communicate with them. Once awareness has been demonstrated and the family has been informed of the results, participants may be invited to participate in further scans, to see if they are capable of communication using the mental imagery task. Participants who are capable of communication can convey information specifically related to their clinical condition (eg, that they are in pain), or regarding other preferences (eg, the entertainment they are provided, or time of day they are fed) which may contribute to their quality of life. ${ }^{29}$

It is possible that a participant might not want others to know that they are aware, in which case disclosure would be morally problematic. If such participants choose not to follow the instructions of researchers, their awareness will remain undetected. Conversely, even if in some cases participants will turn out not to have benefited from others knowing that they are aware, the potential benefits of disclosure are sufficiently high to outweigh potential harms, and justify disclosure; this satisfies the third criterion.

\section{Potential harms and benefits of disclosure to families}

One problematic aspect of the decision to disclose individual results is that the decision is not made by the actual participant. 
While the potential benefits to the participant provide the primary justification for making individual research results available to their families, the potential harms of disclosure are also worthy of consideration. Many families continue to talk to vegetative patients as though they are capable of understanding, and these families would presumably feel vindicated by the disclosure of positive results, and may take comfort in the fact that the participant can hear and comprehend language, and retains the cognitive abilities implied by completion of the mental imagery task. Moreover, just as the participant is likely to benefit from a restored sense of personhood, family members are likely to respond positively to the idea that the participant is aware and capable of continued involvement, at least on some level, in family life.

Of course, it is possible that family members could react badly to news that the participant is, in fact, aware. They may even feel guilty that they have allowed their family member to continue to exist in a state which he or she finds intolerable. ${ }^{30}$ Yet, if the participant is indeed suffering, knowledge of this might allow caregivers to take steps to help make him or her more comfortable; this would be preferable to remaining oblivious to the participant's suffering. Moreover, if a particular family member is acting as the participant's substitute decisionmaker, it is critical that he or she act with the interests of the participant as the foremost concern. Of course, the family may still choose to remain uninformed of the results of the research. (Whether choosing to remain uninformed is consistent with the obligations of a substitute decision-maker is a separate issue, which will not be considered here). Nevertheless, it is the potential benefits to the participant that justify disclosure even if, in some cases, this might be a source of anxiety or distress to the family.

A related issue is the possibility that disclosure might influence the decision to withdraw life-sustaining treatment. In our experience, concerns about end-of-life decision-making are not at the forefront for families of vegetative patients. The participants in question have been in the vegetative state for a median of 5.0 years and the decision to undergo life-sustaining treatment, including tracheostomy and insertion of feeding tubes, is made relatively soon after injury. While a participant's family may eventually decide to withdraw life-sustaining treatment, it is uncommon for these families to withdraw food and fluids.

\section{The participant's family consents to disclosure}

When disclosure of individual research results satisfies the first three criteria, researchers ought to make the results of the mental imagery task available to the participant's family members, who may then consent or decline to have the results disclosed. Whether the family wants to be made aware of results ought to be determined before research is conducted, as part of the informed consent process. Families should also be given the opportunity to revise their decision once research has been concluded. By giving appropriate weight to the decision, researchers demonstrate respect for the participant's family and, by extension, the participant. It is crucial that families understand the possible outcomes of the research so that they are prepared to accept what the results mean, including what can and cannot be inferred about a participant's cognitive capacities. It may also be necessary to correct misconceptions about this research that have been generated by the popular press. ${ }^{31}$ Although family members may choose not to be informed of research findings, we believe that this will not generally be the case. However, if individual family members disagree, then the wishes of the legally designated proxy decision-maker will take precedence. If this individual does not want results disclosed, researchers must comply with his or her wishes. Conversely, if this individual does want results disclosed and other family members do not, the latter may simply choose to remain uninformed.

\section{DISCLOSURE OF NEGATIVE RESULTS}

The obligation to disclose inconclusive or negative individual research results is a more complex issue. Failure to detect awareness can occur for a number of reasons, as mentioned above, and the benefits associated with disclosure of negative results are not easy to discern; for instance, families may misinterpret the fact that no awareness was detected to mean that no awareness is present. There is also the potential that the family may suffer emotional harm (eg, disappointment, anxiety, confusion) if negative results are disclosed.

Nevertheless, there are over-riding moral reasons why researchers should provide these results to families. First and foremost, if the researcher had previously agreed to disclose positive individual results during the informed consent process, failure to do so upon the conclusion of the research will likely be interpreted by families as a negative result. The potential harm may be greater if negative results are not disclosed and the family is left to interpret a lack of results on their own rather than having the matter explained to them by a competent professional. Leaving families to draw their own conclusions suggests a lack of respect and may cause greater anxiety than disclosure of negative results. Available data indicate that research participants want individual research results made available to them even if they are inconclusive or negative. ${ }^{32} 33$ While the desires of participants should not be the sole determinant of policy regarding disclosure (especially with respect to uncertain results), these data suggest that participants find the disclosure of individual research results potentially beneficial. It therefore seems plausible that family members acting on behalf of such participants might derive similar benefit from disclosure.

\section{FUTURE DIRECTIONS}

Having shown that researchers ought to disclose individual research results to participant's families, how should the actual process of disclosure unfold? Although the disclosure of positive results may seem unproblematic, a skilled clinical communicator who is familiar with the research protocol and is able to convey the nuances of the results to each family will be invaluable. Clearly articulating to families the possible outcomes of research before participation will be instrumental in ensuring that families are able to give informed consent not only to participation but also to disclosure. We believe that engaging the families of participants in discussing these issues is critical to maximising the potential benefits of disclosure and minimising the potential harms. Further work is required to develop educational materials for families, and the impact of disclosure of research results on families should be studied systematically.

Of course, family members are not the only ones to whom the research results are relevant. Many of the practical benefits to the participant mentioned above must be implemented by the physician and other staff in charge of care. This means that, in addition to making individual results available to the participant's family, researchers ought to make positive results available to the participant's physician provided that the family agrees. If further research demonstrates that the potential benefits of disclosure to the patient are substantial, it may even be permissible for researchers to disclose positive results to the patient's physician or caregiver when the family declines to be informed. 
Acknowledgements We thank Anthony Belardo for his editorial assistance, and Tommaso Bruni and three anonymous reviewers for their helpful comments.

Contributors MG, CW, AP, LN, DC, DF-E, LG-L and AMO contributed to the conception and design of the project. MG wrote the first draft of the manuscript, and $M G$ and $C W$ led the writing of subsequent versions. All authors commented on sequential drafts and approved the final version.

\section{Competing interests None.}

Provenance and peer review Not commissioned; externally peer reviewed.

Open Access This is an Open Access article distributed in accordance with the Creative Commons Attribution Non Commercial (CC BY-NC 3.0) license, which permits others to distribute, remix, adapt, build upon this work non-commercially, and license their derivative works on different terms, provided the original work is properly cited and the use is non-commercial. See: http://creativecommons.org/ licenses/by-nc/3.0/

\section{REFERENCES}

1 Clayton E, Ross L. Implications of disclosing individual results of clinical research. JAMA 2006;295(1):37.

2 Fernandez $C V$, Kodish E, Weijer C. Informing study participants of research results: An ethical imperative. IRB: Ethics and Human Research 2003;25(3):12-19.

3 Shalowitz D, Miller F. Disclosing individual results of clinical research: implications of respect for participants. JAMA 2005;294(6):737-40.

4 Partridge A, Winer E. Informing clinical trial participants about study results. JAMA 2002;288(3):363-5.

5 Reilly P. When should an investigator share raw data with the subjects? IRB 1980;2:4-5, 12

6 Bardin JC, Voss H, Fins JJ, et al. Dissociations between behavioral and functional magnetic resonance imaging-based evaluations of cognitive function after brain injury. Brain 2011;134(3):769-82.

7 Goldfine A, Victor J, Conte M, et al. Determination of awareness in patients with severe brain injury using EEG power spectral analysis. Clin Neurophysiol 2011;122 (11):2157-68.

8 National Bioethics Advisory Commission. Research involving human biological materials: ethical issues and policy guidance. Rockville, MD: National Bioethics Advisory Commission, 1999.

9 Medical Research Council. Human tissue and biological samples for use in research. Operational and ethical guidelines. London: Medical Research Council, 2001. http:// www.mrc.ac.uk/news-events/publications/human-tissue-and-biological-samples-foruse-in-research/

10 Canadian Institutes of Health Research. CIHR best practices for protecting privacy in health research. Canada: CIHR, 2005.

11 Owen AM. Detecting consciousness: a unique role for neuroimaging. Annu Rev Psychol 2013;64:109-33.

12 The Multi-Society Task Force on PVS. Medical aspects of the persistent vegetative state (1). NEJM 1994;330(21):1499-508.
13 International Working Party. International Working Party: report on the vegetative state. London: Royal Hospital for Neurodisability, 1996.

14 Beaumont JG, Kenealy PM. Incidence and prevalence of the vegetative and minimally conscious states. Neuropsychol Rehabil 2005;15(3-4):184-9.

15 Owen AM, Coleman MR. Functional neuroimaging of the vegetative state. Nature 2008:9:235-43.

16 Schnakers C, Vanhaudenhuyse A, Giacino J, et al. Diagnostic accuracy of the vegetative and minimally conscious state: clinical consensus versus standardized neurobehavioral assessment. BMC Neurol 2009;9(1):35.

17 Andrews $\mathrm{K}$, Murphy L, Munday $\mathrm{R}$, et al. Misdiagnosis of the vegetative state: retrospective study in a rehabilitation unit. BMJ 1996;313(7048):13-16.

18 Childs NL, Mercer WN, Childs HW. Accuracy of diagnosis of persistent vegetative state. Neurology 1993;43(8):1465-7.

19 Monti MM, Vanhaudenhuyse A, Coleman MR, et al. Willful modulation of brain activity in disorders of consciousness. NEJM 2010;362(7):579-89.

20 Boly M, Coleman M, Davis M, et al. When thoughts become action: an fMRI paradigm to study volitional brain activity in non-communicative brain injured patients. Neuroimage 2007;36(3):979-92.

21 Giacino JT, Kalmar K, Whyte J. The JFK coma recovery scale-revised: measurement characteristics and diagnostic utility. Arch Phys Med Rehabil 2004;85(12): 2020-9.

22 Fernández-Espejo D, Owen A. Detecting awareness after severe brain injury. Nat Rev Neurosci 2013;14(1):801-9.

23 Peterson A, Naci L, Weijer C, et al. Communicating with the minimally conscious patient: a principled argument, but not a practical one. Am J Bioeth Neurosci 2013;4(1):52-3.

24 Tresch DD, Sims FH, Duthie EH, et al. Patients in a persistent vegetative state attitudes and reactions of family members. J Am Geriatr Soc 1991;39(1): $17-21$.

25 Owen AM, Coleman MR, Boly M, et al. Response to comments on "Detecting Awareness in the Vegetative State." Science 2007;315(5816):1221.

26 Kitwood T. Dementia reconsidered: the person comes first. Open University Press, 1997.

27 Buber M. I and Thou. New York: Touchstone, 1970.

28 Laureys S, Boly M. What is it like to be vegetative or minimally conscious? Curr Opin Neurol 2007;20:609-13.

29 Peterson A, Naci L, Weijer C, et al. Assessing decision making capacity in the behaviourally non-responsive patient. AJOB Neurosci 2013;4(4):3-14.

30 Kitzinger J, Kitzinger $C$. The 'window of opportunity' for death after severe brain injury: family experiences. Sociol Health IIIn 2013;35(7):1095-112.

31 Samuel G, Kitzinger J. Reporting consciousness in coma: media framing of neuro-scientific research, hope, and the response of families of vegetative and minimally conscious patients. JOMEC J 2013;3:1-23.

32 Shalowitz D, Miller FG. Communicating the results of clinical research to participants: attitudes, practices, and future directions. PLoS Med 2008;5(5):e91.

33 Wendler $D$, Pentz R. How does the collection of genetic test results affect research participants. Am J Med Genet 2007;143A(15):1733-8. 